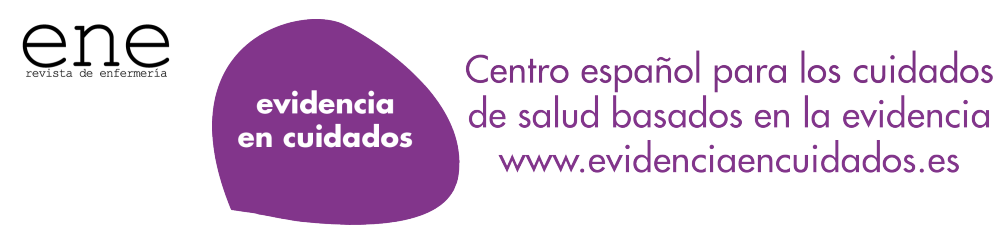

\title{
MANEJO ENFERMERO DEL PACIENTE CON IDEAS SUICIDAS EN EL ÁMBITO EXTRAHOSPITALARIO
}

\author{
AM. Mateo Cervera(1), Ainara Galarza Mateo(2) \\ (1) Complejo Hospitalario de Navarra. Pamplona \\ (2) Centre de Salut Mental Gironés-Pla de l'Estany, Girona
}




\section{RESUMEN}

\section{Introducción.}

El suicidio es un problema de salud pública de índole mundial que va en aumento y cuya gravedad afecta a todos los estratos sociales. En los últimos 45 años esta práctica ha aumentado un $60 \%$ (OMS, 2012), siendo la principal causa externa de muerte en España y la segunda causa principal de defunción en todo el mundo. A nivel Nacional en los últimos datos facilitados por el Instituto Nacional de Estadística se ha recogido un total de 3.569 defunciones en el 2016, siendo más frecuente esta práctica por los varones. Esto supone 10 personas al día en España.

Estos sucesos son asistidos con frecuencia por el servicio de emergencia extrahospitalaria, son el primer eslabón en la actuación frente a conductas suicidas; con frecuencia es el primer contacto que la persona implicada tiene con los servicios sanitarios. Debido a que el ámbito de actuación es la extrahospitalaria, no disponen de los recursos ni facilidades que brinda un ámbito hospitalario, por lo que su actuación deberá estar apoyada por un conocimiento sólido sobre este tipo de paciente para aportar la asistencia eficaz que se busca. En los servicios de emergencias estas habilidades se alcanzan con la práctica diaria y la experiencia, y las guías de práctica clínica indican que "los profesionales sanitarios implicados en la asistencia de pacientes con conducta suicida deberán tener una adecuada formación que les permita evaluar la presencia de factores de riesgo de conducta suicida y registrar el perfil de riesgo del paciente".

El desarrollo de esta revisión se enfoca a identificar y recopilar métodos y recursos comunicativos y no comunicativos que dispone la enfermería en el ámbito extrahospitalario para manejar las conductas descritas.

Objetivo: Saber actuar como enfermera de la emergencia extrahospitalaria en el manejo del paciente con ideas autolíticas.

\section{Métodos, fuentes de datos}

Revisión bibliográfica narrativa de artículos, guías, libros, programas y estudios que desarrollan o analizan aspectos concretos del papel de la enfermería y sus recursos para poder manejar al paciente con ideación suicida. Fuentes de datos consultadas: Web of Science, Medline Plus, Cuiden Plus, PubMed, National guideline clearinghouse (NGC) y Cochrane Library Plus y diferentes páginas web relacionadas con el tema.

\section{Resultados}

Factores de riesgo. No existe una causa única y simple que lleve a esta acción y se considera que el nivel de riesgo aumenta proporcionalmente al número de 
factores presentes. Los menos predecibles asociados a los intentos de suicidios son: edad, sexo, sexualidad, enfermedad somática y estado civil.

Características psicológicas del paciente suicida: Ambivalencia, impulsividad y rigidez.

Para controlar la conducta suicida en el ámbito extrahospitalario lo más importante será:

- Anular el intento afrontando la situación de la forma más eficaz posible, aliviando de inmediato los síntomas y evitando de esta forma la descompensación psicológica.

- La inmediatez que se presente desde los servicios de urgencias y emergencias en ofrecer la ayuda va a influir en el éxito de la misma.

- En una fase posterior se dirigirán las intervenciones al estudio y tratamiento de la causa, así como las medidas de prevención a adoptar en los casos de presencia de factores de riesgo y de tentativa. 


\section{RECOMENDACIONES}

El psicoterapeuta Alejandro Rocamora propone un Modelo de intervención en la crisis AFVA:

- Acoger

- Focalizar el problema

- Valorar la gravedad

- Actuar. Incluye 5 fases:

1. Asegurar la zona, garantizando la seguridad del equipo interviniente, valorando posibilidades de que la persona que amenaza con quitarse la vida también pueda causar daños a terceros. También se apagarán las señales luminosas y acústicas de la ambulancia a medida que nos vayamos acercando al lugar donde se encuentra el sujeto.

2. Al llegar al escenario se realizará una valoración primaria de la persona, otra del entorno, y se tomarán las medidas de seguridad oportunas. Aunque no dispongamos de mucho tiempo, comenzaremos a recoger la mayor cantidad de datos relevantes sobre el suicida a través del entorno que se encuentre en el escenario.

3. Procederemos a establecer contacto con la persona afectada con comunicación verbal y no verbal, no intentaremos hacerlo razonar, solo queremos ganar tiempo para que su estado emocional se transforme. Manifestaremos nuestra intención de ayudar. Seguidamente informaremos de nuestro nombre e intentaremos que nos facilite el suyo. Nos acercaremos al sujeto de forma cautelosa y pidiendo permiso (se puede utilizar de excusa que queremos que nuestro diálogo sea más privado), evitando movimientos bruscos para que no nos perciba como amenaza.

\section{Utilizar herramientas básicas para el acercamiento y para la comunica- ción interpersonal.}

- La empatía con la persona afecta nos permite situarnos ante sus ojos como agentes preocupados y dispuestos a ayudar, con calidez y afecto. También nos facilita el conectar con ella por lo que nos ayuda a interactuar para conseguir nuestros objetivos. La empatía nos implica tomar conciencia de cómo está viviendo el sujeto lo que le ocurre.

- "Escucha activa". Hay una serie de habilidades verbales que empleándolas en los momentos y maneras adecuadas aumentan la relación de acogida y apoyo con la otra persona (rapport), entendido como el entendimiento compartido y empático de las diferentes perspectivas desde las que uno mismo y la otra persona abordan un problema que debe ser solucionado por ambos. 
- El silencio es también una relación de ayuda. Es una habilidad que sin hacerlo explícito combinándolo con un contacto físico adecuado, una mirada y/o un gesto puede transmitir que se "está ahí". Este puede ser más potente que un discurso lleno de elocuencia, ya que para esa persona puede que suene vacío.

5. Interacción verbal. Cuanto más tiempo estemos interactuando, más tiempo tendremos para que alivie sus sentimientos y preocupaciones, se desahogue y tranquilice y pueda bajar la presión psíquica y nivel de ansiedad que tiene. Siempre dejaremos que hable o se exprese la persona antes que realizar nuestras preguntas. La técnica del parafraseo nos abre la puerta a que el sujeto se exprese, repitiendo la última frase que diga en tono de pregunta. Utilizaremos preguntas cortas y directas, evitando la sobreutilización. Empezar por lo general e inocuo e ir profundizado a medida que nos vaya dejando hacia nuestra pregunta clave: ¿por qué quiere realizar esta conducta?

Finalmente, debería ser trasladado en una UVI móvil a un centro hospitalario, y que debería hacerlo sedado. Si el paciente no va sedado irá en un Soporte Vital Básico (SVB), siempre acompañado por un profesional y vigilado de manera constante hasta el final del traslado.

\section{Comentario}

La Organización Mundial de la Salud (OMS), ante el tema del suicido, insiste en actuar en cuatro líneas de acción: 1. Tomar conciencia sobre la magnitud del suicidio como problema de salud pública mundial; 2. Informar sobre lo qué podemos hacer para prevenirlo; 3. Combatir el estigma asociado con el suicidio; 4. Hacer saber a las personas que están pasando por momentos difíciles que no están solas1.

Así mismo, la OMS considera el suicidio y la tentativa de suicidio como un problema de salud pública y es uno de los factores más importantes de morbilidad en jóvenes: ocupa la segunda causa de muerte en el mundo entre jóvenes de 15 a 29 años. Se cree que las dificultades de afrontamiento y resolución de problemas son algunos de los factores más relevantes 1 .

Las conductas suicidas son el iceberg de un sufrimiento personal y social de grandes dimensiones, que afecta amplios sectores de población. La prevención del suicidio no es una tarea fácil debido a las múltiples connotaciones que contiene de tipo filosófico, cultural, social, psicológico y médico. Con todo, el suicidio y su abordaje afectan a toda la sociedad: los modelos de vida y de relación, así como al sistema de valores. La proporción de suicidios evitable a través de acciones preventivas es pequeña, pero es un deber social y sanitario llevar a cabo acciones para alcanzar los objetivos de prevención posibles2. 
El número de muertes por suicidio ha alcanzado los 800.000 en el mundo, y el número de intentos puede ser alrededor de 20 veces superior al de fallecimientos. Muchos de los estudios indican que es prevenible si se interviene de manera oportuna y a tiempo con estrategias multisectoriales e integrales. La ideación suicida y el intento son más frecuentes en la población femenina y el suicidio consumado en la masculina ${ }^{1}$.

El artículo hace una revisión de la actuación de los servicios de salud extra hospitalarios ante una llamada por intento de suicido. Es escasa la literatura publicada sobre la actuación individual del personal de enfermería, ya que el abordaje en el momento concreto de la crisis debe ser multidisciplinar. Las recomendaciones propuestas en el artículo pueden ser aplicadas por el personal del equipo de atención, siendo la posibilidad de éxito directamente proporcional a la formación y experiencia del equipo que atiende la llamada.

El plan de actuación posterior ha de contemplar los vínculos con el entorno del paciente y sus redes de apoyo y la familia. En la atención posterior al intento, la Atención Primaria (AP) sanitaria y la social también han de tener un papel relevante. El vínculo existente entre profesional y paciente es un facilitador del seguimiento y tratamiento. El paciente puede considerar más cercana la intervención de los profesionales habituales que la de los servicios especializados. Sin patología mental definida se puede producir un rechazo de la atención psiquiátrica. La resistencia a la atención especializada también se puede dar en personas con enfermedad mental y en estos casos el vínculo con la AP acostumbra a ser el recurso asistencial de continuidad2.

La enfermera tiene un papel fundamental en la detección precoz de la ideación suicida debido a su presencia transversal en los servicios sanitarios. Cualquier comentario referente, directa o indirectamente, a la muerte debe ser tomado en serio, llevar a cabo una valoración del riesgo de suicidio, documentar la valoración, comentar la valoración con otros miembros del equipo de salud que atienden al paciente y crear un plan para la seguridad y el cuidado según lo determinado en el resultado de la valoración ${ }^{3}$.

En España las cifras son preocupantes, ya que el problema afecta muy especialmente apersonas jóvenes. Las acciones y las ideaciones suicidas tienen un carácter multifactorial; esto afecta a todo el núcleo familiar y su contexto. Los jóvenes que cuentan con menos herramientas para afrontar y resolver los problemas y menos tolerancia a la frustración son más susceptibles a presentar el fenómeno. El suicidio tiene diferentes implicaciones socioculturales y sociopolíticas y es un proceso que inicia con la ideación suicida, y el intentarlo ya se convierte en un factor de riesgo importante para consumarlo4.

El Ministerio de Salud está trabajando en una línea estratégica que permita el abordaje integral de la prevención de la conducta suicida en España, dentro de la Estrategia de Salud Mental 2019-2024. 
"La proporción de suicidios evitable a través de acciones preventivas es pequeña, pero es un deber social y sanitario llevar a cabo acciones para alcanzar los objetivos de prevención posibles. ’3

\section{REFERENCIAS}

1. Prevención del suicidio. En: Organización Mundial de la Salud [en línea] [consultado el 12/12/2019]. Disponible en https://www.who.int/es/news-room/fact-sheets/detail/suicide

2. Fernández de Sanmamed MJ, García J, Mazo MV, Mendive JM, Serrano E, Zapater F. Consideraciones para un abordaje social y sanitario del suicidio a propósito del Código Riesgo de Suicidio. Barcelona: Fòrum Català d'Atenció Primària. 2018

3. GBP RNAO: Valoración y cuidado de los adultos en riesgo de ideación y comportamiento suicida. [en línea] [consultado el 12/12/2019]. Disponible en http://www.evidenciaencuidados.es/es/attachments/article/ 46/D0026_BPG_Suicidio_2009.pdf

4. Cañón Buitrago SC, Carmona Parra JA. Ideación y conductas suicidas en adolescentes y jóvenes. Rev Pediatr Aten Primaria. 2018;20:387-97. 